\title{
Singularity Analysis of a three translational degrees of freedom Parallel Mechanism
}

\author{
Binjiu Yang ${ }^{1, a^{*}, \text { Xiu Qin }}{ }^{2, b}$ \\ ${ }^{1}$ Harbin Institute of Technology at Weihai, china \\ ${ }^{2}$ Harbin Institute of Technology at Weihai, china \\ ayangbinjiu@163.coml, b qinxiu19870414@126.com,
}

Keywords: parallel machanism; Jacobian matrix; singular configuration

Abstract. The singularity of a new 3-DOF parallel mechanism was analyzed. The position equation of the mechanism was gotten by the inverse kinematics solution. The velocity equation of the machine was gotten by the derivation of the position equation, and then got the Jacobian matrix, and the singularity conditions were analyzed. The singularity analysis will lay the trajectory planning of the mechanism.

\section{Introduction}

A parallel mechanism, because of its stiffness, high precision, no accumulating position error, has become a hot research in the mechanical field. At present, in the aviation, aerospace, submarine operations, manufacturing, allied health and micro-electromechanical systems, the parallel institutions have a wide and important application.

For the parallel mechanism, the singularity usually refers to the special nature of the platform when it access to the local instantaneous degree of freedom, for example, transient loss of body rigidity and reduce of positioning accuracy of the end, driving force can not balance the specific generalized forces of the moving platform, and many other negative factors. At this point, the configuration is defined as the singularity. When the body is in the singularity, it will be out of control. Therefore, how to predict the distribution of the singularity within the workspace is a very crucial problem at the design stage. And obtaining the distribution of singularity will help to correct the trajectory of planning agencies, precise control of the output power or moment. The singularity analysis of parallel institutions mainly from the Jacobian matrix[1].

\section{Institutions introduction}

This article mainly introduces the singularity analysis of the 3-PTT parallel mechanism; the structure is shown in Figure 1.



Fig.1. Structure diagram and coordinate system

1-slider; 2-guide rail; 3-parallel institution; 4-moving platform; 5-universal joint; 6-pole set 
As shown in Figure 1, three rails evenly distributed into $120^{\circ}$ in the fixed platform. The fixed platform and the campaign connected by three branched-chains, and each chain composed by the slide, set pole and hinge. The slider, achieved transmission through the screw pair with the rail on the fixed platform, connected by universal joints with the fixed length poles. The poles connected with the moving platform through universal joints. The motor drove the screw rotation, the screw drove the slider translating along the rail, and the slider made the poles move through universal joints, and the travel of poles put the moving platform in motion. As one of the branched-chains used five-bar in space, limiting two rotational degrees of freedom of the motion platform, the other two branched-chains limited a rotational DOF, so in theory, the motion platform has only three translational degrees of freedom. The following calculated the DOF of the mechanism through mathematical method.

The number of needed independent variables for determining the body position is called degree of freedom. DOF is resolved by the number of members and joints, and the constraint condition. Currently, the number of spatial DOF is calculated by

$$
M=d(n-q-1)+\sum_{i=1}^{q} f_{i}
$$

Where $\mathrm{d}$ is the rank of the machanism, and $d=6-\lambda, \lambda$ is the number of public sector constraints, which is the number of lost degrees of freedom of all components; $n$ is the number of components for the body; $q$ is the number of joint; $f_{i}$ is the DOF for the I joint.

The calculation of DOF for the 3-PTT horizontal sliders type parallel mechanism is as follows: with the five-bar shown in figure 1 as a whole, there is eight components in the body, $n=8$ (three sliders, three poles, one fixed platform, one motion platform), nine joints, $q=9$ (three prismatic pairs, six universal joints), and each prismatic pair has one freedom, each universal joint has two freedoms, so $\sum_{i=1}^{q} f_{i}=3+2 \times 6=15$, the DOF for the mechanism is $M=d(n-q-1)+\sum_{i=1}^{q} f_{i}=6(8-9-1)+15=3$, have the same DOF with the theoretical analysis.

\section{Inverse kinematics}

Established coordinate system shown in Figure 1, the fixed coordinate system O-xyz in the fixed platform, $\mathrm{x}$-axis coincides with the axis of the third guide rail, $\mathrm{z}$-axis perpendicular to the plane, which the three rails are in; the dynamic coordinate O'-x'y'z' in the moving platform, where x'//x, $y^{\prime} / / y, z^{\prime} / / z . S_{1} 、 S_{2} 、 S_{3}$ respectively the displacement of three sliders.

As the third chain only limited two rotational degrees of freedom, so in the kinematics calculation, it can take the center of the triangle formed by the three joints on the fixed and moving platform $\mathrm{P}_{3}$ 、 $\mathrm{B}_{3}$. So the coordinates of $\mathrm{P}_{1} 、 \mathrm{P}_{2} 、 \mathrm{P}_{3}$ in the fixed coordinate-system $\{\mathrm{P}\}: \mathrm{O}$-xyz are $\mathrm{P}_{1}$ $\left(-\frac{1}{2} S_{1}, \frac{\sqrt{3}}{2} S_{1}, 0\right), \mathrm{P}_{2}\left(-\frac{1}{2} S_{2},-\frac{\sqrt{3}}{2} S_{2}, 0\right), \mathrm{P}_{3}\left(S_{3}, 0,0\right) ;$ the coordinates of $\mathrm{B}_{1} 、 \mathrm{~B}_{2} 、 \mathrm{~B}_{3}$ in the dynamic coordinate-system $\{\mathrm{B}\}: \mathrm{O}^{\prime}-\mathrm{x}^{\prime} \mathrm{y}^{\prime} \mathrm{z}^{\prime}$ are $\mathrm{B}_{1}\left(-\frac{1}{2} R, \frac{\sqrt{3}}{2} R, 0\right), \mathrm{B}_{2}\left(-\frac{1}{2} R,-\frac{\sqrt{3}}{2} R, 0\right), \mathrm{B}_{3}(R, 0,0)$, where $\mathrm{R}$ is the radius of the moving platform(radius of circumcircle formed by $\mathrm{B}_{1} 、 \mathrm{~B}_{2} 、 \mathrm{~B}_{3}$ ).Expressed $\mathrm{B}_{\mathrm{i}}(\mathrm{i}=1,2,3)$ in the fixed coordinate-system: ${ }_{B_{i}}={ }_{B}^{P} R \cdot{ }_{B}^{B}{ }_{B}+{ }_{P} \stackrel{W u}{P}_{B O}$, where ${ }_{B}^{P} R$ is the rotation matrix $\{\mathrm{B}\}$ relative $\{\mathrm{P}\}$, as there are only three translate DOF, ${ }_{B}^{P} R=\left[\begin{array}{lll}1 & 0 & 0 \\ 0 & 1 & 0 \\ 0 & 0 & 1\end{array}\right]$, so ${ }_{B_{i}}^{\mathbf{u n}}={ }_{B}^{{ }_{B} \mathbf{U n}}{ }_{B_{i}}+{ }_{P}{ }_{P} P_{B O}$.If 
$P_{B O}=\left(x^{\prime}, y^{\prime}, z^{\prime}\right), \mathrm{B}_{\mathrm{i}}$ can be expressed in the fixed coordinate-system as

$B_{1}\left(x^{\prime}-\frac{1}{2} R, y^{\prime}+\frac{\sqrt{3}}{2} R, z^{\prime}\right), B_{2}\left(x^{\prime}-\frac{1}{2} R, y^{\prime}-\frac{\sqrt{3}}{2} R, z^{\prime}\right), B_{3}\left(x^{\prime}+R, y^{\prime}, z^{\prime}\right)$.It can be seen from the geometry that $l_{i}={\stackrel{P}{i} B_{i}}_{\text {,so }}$

$\left\{\begin{array}{l}\left(x^{\prime}-\frac{1}{2} R+\frac{1}{2} S_{1}\right)^{2}+\left(y^{\prime}+\frac{\sqrt{3}}{2} R-\frac{\sqrt{3}}{2} S_{1}\right)^{2}+\left(z^{\prime}\right)^{2}=l_{1}^{2} \\ \left(x^{\prime}-\frac{1}{2} R+\frac{1}{2} S_{2}\right)^{2}+\left(y^{\prime}-R \frac{\sqrt{3}}{2}+\frac{\sqrt{3}}{2} S_{2}\right)^{2}+\left(z^{\prime}\right)^{2}=l_{2}^{2} \\ \left(x^{\prime}+R-S_{3}\right)^{2}+\left(y^{\prime}\right)^{2}+\left(z^{\prime}\right)^{2}=l_{3}^{2}\end{array}\right.$

The inverse kinematics solution obtained

$$
\left\{\begin{aligned}
S_{1}= & -R-\frac{\sqrt{3}}{2} y^{\prime}+\frac{1}{2} x^{\prime} \\
& +\frac{1}{2} \sqrt{-y^{\prime 2}-2 \sqrt{3} x^{\prime} y^{\prime}-3 x^{\prime 2}-4 z^{\prime 2}+4 l_{1}^{2}} \\
S_{2}= & R+\frac{\sqrt{3}}{2} y^{\prime}+\frac{1}{2} x^{\prime} \\
& +\frac{1}{2} \sqrt{-y^{\prime 2}+2 \sqrt{3} x^{\prime} y^{\prime}-3 x^{\prime 2}-4 z^{\prime 2}+4 l_{2}^{2}} \\
S_{3}= & x^{\prime}+R+\sqrt{-y^{\prime 2}-z^{\prime 2}+l_{3}^{2}}
\end{aligned}\right.
$$

Calculation of the Jacobian matrix

Jacobian matrix for parallel mechanism shows the linear relationship between input and output velocity. The following used Jacobian matrix for the singularity[2,3].

Draw the side view for one of the chains, shown in Figure 2

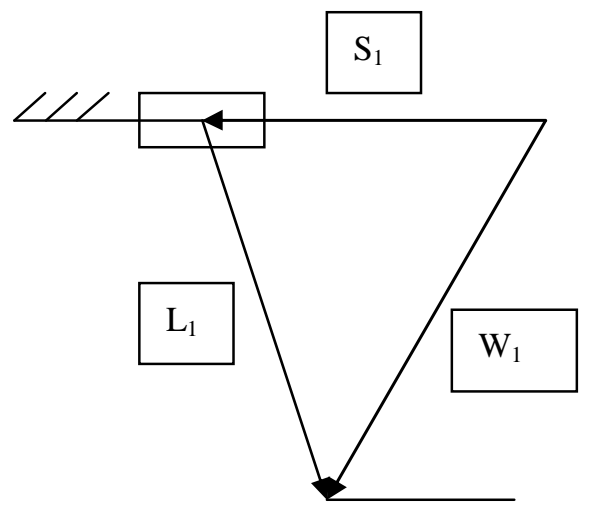

Fig.2. Side view of a branched-chain

Where $\mathrm{S}$ is the displacement of slider, $\mathrm{L}$ is the length of link, $\mathrm{W}$ is the pose of joint on the moving platform expressed in the fixed coordinate-system.

Expressed equation (3) in vector as

$$
S_{i}=e_{i} \cdot W_{i}+\left[\left(e_{i} \cdot W_{i}\right)^{2}-\left(W_{i}^{2}-L_{i}^{2}\right)\right]^{\frac{1}{2}}
$$


The Jacobian matrix of the manipulator had been derived by taking the derivative of equation (4)

$S_{i} \dot{S}_{i}+W_{i} \cdot \dot{W}_{i}-\dot{S}_{i} \cdot e_{i} \cdot W_{i}-S_{i} e_{i} \cdot W_{i}=0 \quad i=1,2,3$

The solution is

$\dot{S}_{i}=\frac{S_{i} \cdot e_{i}-W_{i}}{S_{i}-e_{i} \cdot W_{i}} \dot{W}_{i} \quad i=1,2,3$

(6)

Where $\dot{W}_{i}$ is the velocity vector of the joint on the moving platform, $\dot{S}_{i}$ is the input velocity. The motion platform only do the translation, so the velocity of the platform center $\mathrm{O}$ ' is equal with the joints, that is $\dot{W}_{i}=V_{o^{\prime}}$,so equation (6) can be expressed as

$\dot{S_{i}}=\frac{S_{i} \cdot e_{i}-W_{i}}{S_{i}-e_{i} \cdot W_{i}} V_{o^{\prime}}$

Expressed equation (7) in matrix form as

$$
\left[\begin{array}{c}
\dot{S}_{1} \\
\dot{S}_{2} \\
\dot{S}_{3}
\end{array}\right]=\left[\begin{array}{l}
\frac{S_{1} \cdot e_{1}-W_{1}}{S_{1}-e_{1} \cdot W_{1}} \\
\frac{S_{2} \cdot e_{2}-W_{2}}{S_{2}-e_{2} \cdot W_{2}} \\
\frac{S_{3} \cdot e_{3}-W_{3}}{S_{3}-e_{3} \cdot W_{3}}
\end{array}\right]^{T}\left[\begin{array}{l}
V_{o^{\prime} x} \\
V_{o^{\prime} y} \\
V_{o^{\prime} z}
\end{array}\right]
$$

where $V_{o^{\prime} x}, V_{o^{\prime} y}, V_{o^{\prime} z}$ are velocity components in three directions. So the Jacobian matrix for the mechanism is

$$
J=\left[\begin{array}{c}
\frac{S_{1} \cdot e_{1}-W_{1}}{S_{1}-e_{1} \cdot W_{1}} \\
\frac{S_{2} \cdot e_{2}-W_{2}}{S_{2}-e_{2} \cdot W_{2}} \\
\frac{S_{3} \cdot e_{3}-W_{3}}{S_{3}-e_{3} \cdot W_{3}}
\end{array}\right]^{T}
$$

\section{Singularity Analysis}

Put the coordinates of vectors into equation (9)

$$
J=\left[\begin{array}{ccc}
\frac{x^{\prime}-\frac{1}{2} R+\frac{1}{2} S_{1}}{z^{\prime}} & \frac{y^{\prime}+\frac{\sqrt{3}}{2} R-\frac{\sqrt{3}}{2} S_{1}}{z^{\prime}} & 1 \\
\frac{x^{\prime}-\frac{1}{2} R+\frac{1}{2} S_{2}}{z^{\prime}} & \frac{y^{\prime}-\frac{\sqrt{3}}{2} R+\frac{\sqrt{3}}{2} S_{2}}{z^{\prime}+S_{3}} & 1 \\
\frac{y^{\prime}}{z^{\prime}} & 1
\end{array}\right]
$$

So the Jacobian determinant is 


$$
\operatorname{det}(J)=\frac{1}{z^{\prime 2}}\left|\begin{array}{ccc}
x^{\prime}-\frac{1}{2} R+\frac{1}{2} S_{1} & y^{\prime}+\frac{\sqrt{3}}{2} R-\frac{\sqrt{3}}{2} S_{1} & 1 \\
x^{\prime}-\frac{1}{2} R+\frac{1}{2} S_{2} & y^{\prime}-\frac{\sqrt{3}}{2} R+\frac{\sqrt{3}}{2} S_{2} & 1 \\
x^{\prime}+R-S_{3} & y^{\prime} & 1
\end{array}\right|
$$

$$
=\frac{1}{z^{\prime 2}}\left|\begin{array}{ccc}
x^{\prime}-\frac{1}{2} R+\frac{1}{2} S_{1} & y^{\prime}+\frac{\sqrt{3}}{2} R-\frac{\sqrt{3}}{2} S_{1} & 1 \\
\frac{1}{2} S_{2}-\frac{1}{2} S_{1} & -\sqrt{3} R+\frac{\sqrt{3}}{2} S_{2}+\frac{\sqrt{3}}{2} S_{1} & 0 \\
\frac{\sqrt{3}\left(S_{2}-S_{1}\right)-\left(3 R-2 S_{3}-S_{2}\right)\left(-2 \sqrt{3} R+\sqrt{3} S_{2}+\sqrt{3} S_{1}\right)}{2 \sqrt{3}\left(R-S_{2}\right)} & 0 & 0
\end{array}\right|
$$

(11)

It can be seen from equation (11) that, when $S_{1}=S_{2}=S_{3},|J|=0$ the institution is in the singular position. Moreover, $S_{2} \neq R$, considering its symmetry, it indicate that the three branched-chain can not in a vertical position at the same time.

\section{References}

[1] Yonggang Cao, Yuru Zhang.Singularity analysis of 6-RSS-type parallel mechanism. Mechanical Engineering, 2008,44(6):79 86

[2] Jigang Chen, Xinjun Liu.Singularity analysis for a new 3-DOF parallel mechanism. Machine Design and Research.2004,20(4):22 25

[3] Zonggang Xu, Xiuqing Hao, Min Dong, Kaiyun Cai, Kejie Wang. Singularity analysis for 3-PCR parallel mechanism. Shandong University of Technology(Natural Science),2009,23(1):17 20 